\title{
Longitudinal Outbreak of Multidrug-Resistant Tuberculosis in a Hospital Setting, Serbia
}

\author{
Irena Arandjelović, ${ }^{1}$ Matthias Merker, ${ }^{1}$ \\ Elvira Richter, Thomas A. Kohl, \\ Branislava Savić, Ivan Soldatović, Thierry Wirth, \\ Dragana Vuković,, ${ }^{1}$ Stefan Niemann ${ }^{1}$
}

A retrospective population-based molecular epidemiologic study of multidrug-resistant Mycobacterium tuberculosis complex strains in Serbia (2008-2014) revealed an outbreak of TUR genotype strains in a psychiatric hospital starting around 1990. Drug unavailability, poor infection control, and schizophrenia likely fueled acquisition of additional resistance and bacterial fitness-related mutations over 2 decades.

$\mathrm{T}$ he overall burden of tuberculosis (TB) in Serbia has been greatly reduced in recent years $(1,2)$. However, a recent study revealed transmission of multidrug-resistant (MDR) Mycobacterium tuberculosis complex (MTBC) strains (i.e., MTBC strains resistant to at least rifampin and isoniazid) in Belgrade (3). In addition, data retrieved from the national database of MDR TB patients indicate a concentrated burden of MDR TB and extensively drug-resistant (XDR) TB, defined as additional resistance to 1 fluoroquinolone and 1 of the 3 injectable second-line drugs, among psychiatric inpatients in Serbia. To gain more insights into countrywide transmission routes, strain dynamics, and bacterial evolution over time, we retrospectively investigated all $(\mathrm{n}=110)$ patients who received a diagnosis of MDR TB during January 1, 2008-May 31, 2014, in Serbia.

\section{The Study}

We subjected 1 MTBC isolate per patient to phenotypic drug susceptibility testing and whole-genome sequencing (WGS) (Appendix 1, https://wwwnc.cdc.gov/EID/ article/25/3/18-1220-App1.pdf). We retrieved patients' demographic, epidemiologic, and clinical data from the

\footnotetext{
Author affiliations: University of Belgrade, Belgrade, Serbia

(I. Arandjelović, B. Savić, I. Soldatović, D. Vuković);

Leibniz-Zentrum für Medizin und Biowissenschaften, Borstel,

Germany (M. Merker, T.A. Kohl, S. Niemann); Laboratory

Limbach, Heidelberg, Germany (E. Richter); Paris Sciences \&

Lettres University, Paris, France (T. Wirth); Sorbonne Universités,

Paris (T. Wirth); German Center for Infection Research, Borstel,

Germany (S. Niemann)
}

DOI: https://doi.org/10.3201/eid2503.181220 national database of MDR TB patients, as well as from their medical and laboratory records.

Most patients were male $(87 / 110,79.1 \%)$ and born in Serbia (107/110, 97.3\%); mean age was 49.5 years (range 15-83). We observed concurrent conditions for 55 patients; schizophrenia was the most prevalent $(26 / 55,47.3 \%)$. Of the 110 patients, $61(55.5 \%)$ had previously experienced TB. Susceptibility testing results showed that $19 / 110$ (17.3\%) MDR MTBC isolates were resistant to all first-line drugs, and 11/110 (10.0\%) were classified as XDR (Appendix 1 Table 1). We successfully completed WGS for 103/110 isolates, representing $93.6 \%$ of all MDR TB cases recorded over the study period.

We considered 6,512 single-nucleotide polymorphisms (SNPs) differentiating all isolates to analyze their phylogenetic relationships. The MDR MTBC strain population comprised 37/103 (35.9\%) isolates classified as lineage 4.2.2.1 (TUR genotype), 20/103 (19.4\%) isolates of lineage 4.1.2 (Haarlem genotype), 17/103 (16.5\%) isolates of lineage 2.2.1 (Beijing genotype), 15/103 (14.6\%) isolates of lineage 4.8 (H37Rv-like strains), 8/103 (7.8\%) isolates of lineage 4.4.1.1 (S-type), 2/103 (1.9\%) isolates of lineage 4.2.1 (URAL genotype), 1 isolate of lineage 4.1 (Ghana), and 1 nonclassified lineage 4 isolate. Among lineage 2.2.1 Beijing isolates, the previously described Europe/Russia W148 MDR outbreak isolates (4) were most prevalent, present in 14/17 (82.4\%) of the cases (Figure 1; Appendix 2, https:// wwwnc.cdc.gov/EID/article/25/3/18-1220-App2.xls).

Seeking to identify recent chains of transmission, we defined molecular clusters as surrogate markers for epidemiologically linked cases (Appendix 1). Overall, 63/103 (61.2\%) isolates could be assigned to 12 different clusters, each including 2-17 patients. The 2 largest clusters, 1 containing 14 and 1 containing 17 cases, comprised isolates of TUR genotype; the next-largest cluster was of 7 Beijing Europe/Russia W148 isolates. For all 63 suggested epidemiologic links, we were able to retrospectively identify $40(63.5 \%)$ epidemiologic links (e.g., household and social contacts) (Appendix 1 Figure 1).

Our main finding was that $35 / 37$ (94.6\%) TUR isolates shared identical mutations that confer drug resistance to isoniazid ( $k a t G \mathrm{~S} 315 \mathrm{~T}$ ), streptomycin (rpsL K43R), and ethambutol (embB Q497R); we therefore classified them as TUR-outbreak isolates. TUR-outbreakisolates furtherdifferentiated into 2 individual transmission chains characterized

${ }^{1}$ These authors contributed equally to this article. 


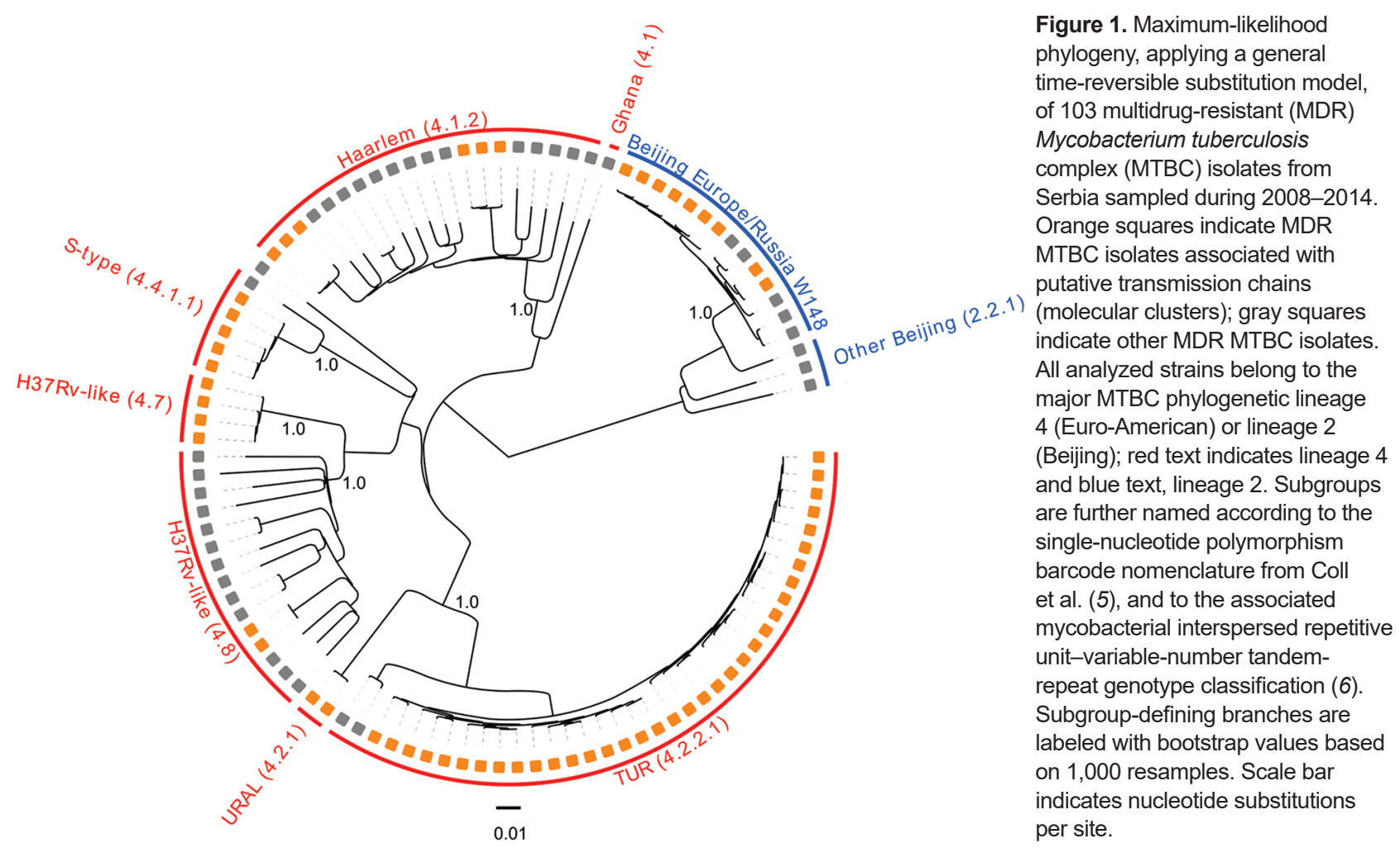

by 2 distinct rifampin resistance-mediating mutations: rроB S450W in 1998 (95\% highest posterior density [HPD] 1993-2001) and rрoB S450L in 2003 (95\% HPD 2000-2005) (Figure 2, panel A; Appendix 1 Figure 2). Subsequently, both strain populations acquired individual mutations in other RNA polymerase genes (rpoA P25R, rpoC $\mathrm{V} 431 \mathrm{M}$, and $r p o C \mathrm{~F} 452 \mathrm{~L}$ ), which have been proposed to enhance the in vitro growth rate of rifampin-resistant strains (7). Furthermore, rpoA mutations in the entire dataset were more likely to arise in clustered isolates than in unique isolates $(20 / 63$ vs. $1 / 40 ; p<0.001)$, thus indicating their ability to restore fitness of $r p o B$ mutants, increase transmission success, or both.

Of the 35 TUR-outbreak isolates, 26 (74.3\%) were from patients hospitalized in Bela Crkva (BC) Hospital, the national center for treatment of all psychiatric patients with concomitant respiratory illnesses. Of note, 22 (84.6\%) of these 26 patients had been transferred from 7 different psychiatric hospitals to BC Hospital for pulmonary diagnosis and treatment; 5 were admitted at BC Hospital with either confirmed or suspected TB diagnosis (Appendix 2). Screening for TB at time of admission had not been implemented in BC Hospital during the study period.

To determine the geographic origin of the 3 -fold resistant TUR ancestor and to test for the putative independent introduction of 2 different rifampin-resistant cases to the BC Hospital from other hospitals, we extended our Bayesian approach with a discrete trait model introducing the likely place of infection for each patient. We used 2 assumptions: first, a fast disease progression assumed infection and diagnosis of MDR TB within the first 2 years after admission to BC Hospital; and second, a slow disease progression in which patients who received a diagnosis within 2 years after admission were identified as latent MDR TB cases, meaning they had contracted the infection in their hometown or a previous hospital.

The comparison of both models using path sampling clearly favored the fast progression model, suggesting the origin of the TUR outbreak in BC Hospital with a probability of $53 \%$ (i.e., node location probability; second likely origin was Belgrade, 12\%) (Figure 2, panel A). The 2 unique rifampin-resistance mediating mutations were also more likely to have originated in BC Hospital itself $(51 \%$ for rpoB S540W node, $95 \%$ for rpoB S540L node, and $<15 \%$ for other location probabilities). Individual transmission events occurred in remote cities but also within Belgrade (Figure 2, panels A, B). In comparison, applying the slow TB progression hypothesis, TUR outbreak strains would have been imported multiple times from different regions throughout the country to BC Hospital, with node location probabilities $\leq 10 \%$ for all locations (Appendix 1 Figure 3). Tracing the time of hospitalization at BC Hospital and MDR TB diagnosis of patients infected with TUR strains backward revealed that the 2 clades (defined by rроB $\mathrm{S} 450 \mathrm{~L}$ and $r p o B \mathrm{~S} 450 \mathrm{~W}$ ) indeed coexisted over 2 decades (Appendix 1 Figure 4). 

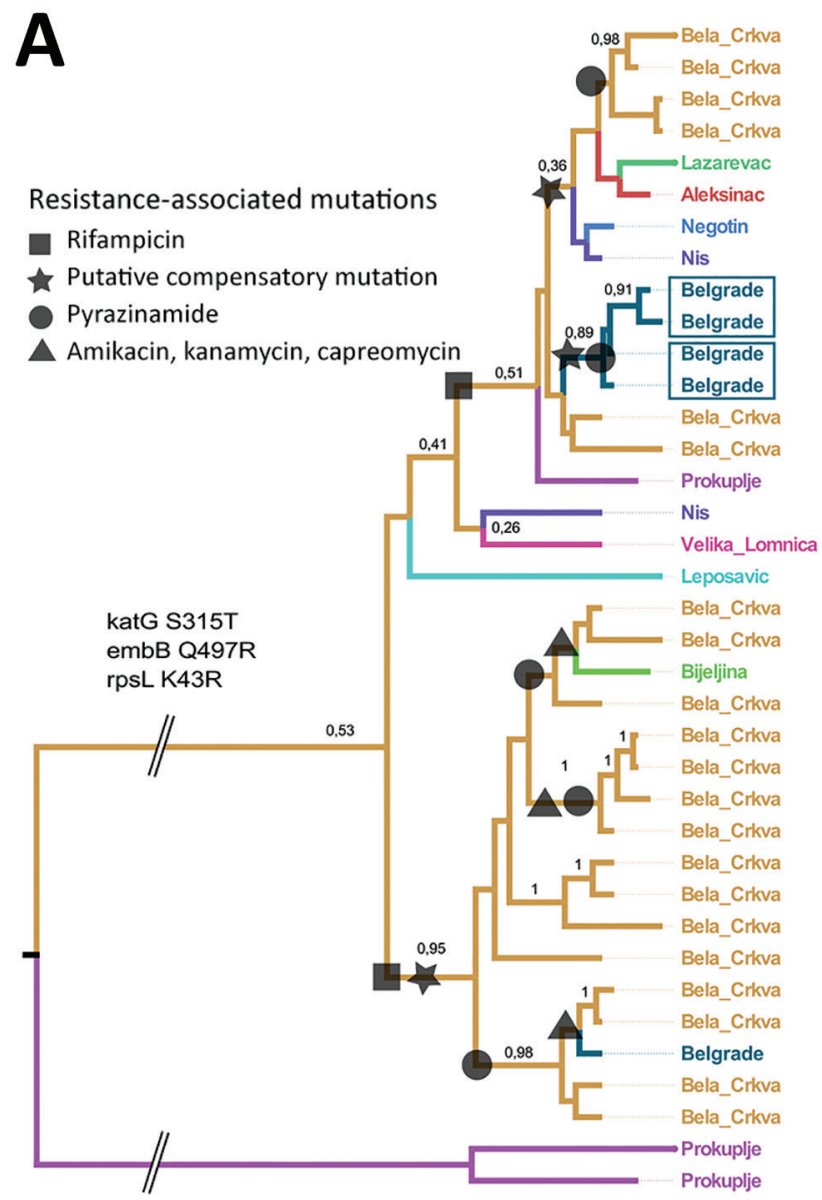

20 years

Figure 2. Most likely temporal and spatial origin of Mycobacterium tuberculosis complex (MTBC) TUR genotype outbreak strains in Serbia. A) Location annotated time-scaled phylogeny (maximum clade credibility tree) derived from a Bayesian discrete trait phylogeographical analysis of 37 lineage 4.2.2.1 (TUR genotype) multidrug-resistant (MDR) MTBC isolates. Branches are colorcoded according to the most likely place of infection, assuming a fast-progression hypothesis (Appendix 1, https://wwwnc.cdc.gov/ EID/article/25/3/18-1220-App1.pdf). Branches are annotated with location probabilities; symbols represent acquisition of individual resistance-related mutations shared by all derived strains. B) Regional and countrywide spread of individual TUR genotype outbreak strains originating from Bela Crkva Hospital. Arrows indicate inferred location changes determined from the genealogy shown in panel A.

\section{Conclusions}

In a retrospective approach using WGS-based molecular epidemiology, Bayesian statistics, and detailed epidemiologic investigations, we show that MDR TB in Serbia is associated with nosocomial transmission at BC Hospital, likely accompanied by a fast progression to disease within 2 years. Drug unavailability in the 1990s (8), schizophrenia as a recognized cause of unsuccessful completion of TB treatment (9), and long-term and repeated hospitalizations under extremely adverse living conditions (10), together with the absence of a TB infection control program, are believed to be the main drivers of the evolutionary trajectories and success of TUR-outbreak strains in Serbia. The TUR-outbreak strain was considered intrinsically resistant to 3 first-line drugs and probably acquired an MDR

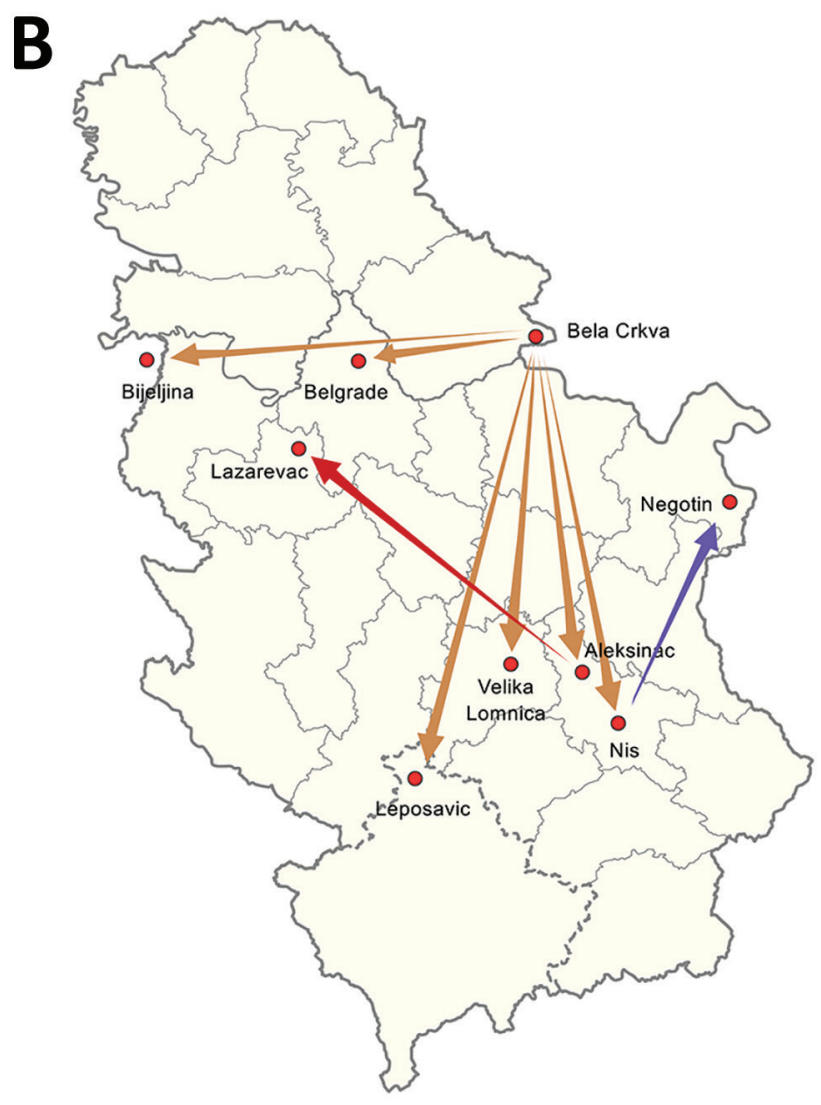

genotype in 2 independent events in $\mathrm{BC}$ Hospital during the 1990s. Subsequently, putative compensatory mechanisms were selected, the strain acquired individual XDR genotypes, and it spread into other settings in Serbia by family contacts and other modes.

Detection of the extensive transmission network in BC Hospital led to the development and implementation of an appropriate TB infection control program featuring the use of rapid laboratory tests for prompt detection of new cases, completion of appropriate second-line treatment regimens, and markedly expanded contact tracing activities. Since 2015, only 1 new case of MDR TB has been recorded in BC Hospital. However, MDR TB transmission in the general population must continue to be carefully monitored. 


\section{Acknowledgments}

We thank J. Zallet, I. Razio, and V. Mohr for excellent support in library preparation and whole-genome sequencing. We thank R. Ćurčić for her help in retrieving data from the Serbian National Database of MDR TB patients, and Lj. Ušan-Miličković for providing data on patients hospitalized in Bela Crkva Hospital. We gratefully acknowledge the Serbian Laboratory Network for tuberculosis and the Supranational Reference Laboratory for Tuberculosis, Forschungszentrum Borstel, Borstel, Germany, for contributions to the study.

\section{About the Author}

Dr. Arandjelović is a teaching assistant in the Department of Microbiology, Faculty of Medicine, University of Belgrade, Belgrade. Her primary research interests are mycobacteriology, drug resistance, and molecular epidemiology of tuberculosis.

\section{References}

1. World Health Organization. Global tuberculosis report. Contract no.: WHO/HTM/TB/2017.23. Geneva: The Organization; 2017.

2. European Centre for Disease Prevention and Control; WHO Regional Office for Europe. Tuberculosis surveillance and monitoring in Europe 2017. Stockholm: European Centre for Disease Prevention and Control; 2017.

3. Vuković D, Rüsch-Gerdes S, Savić B, Niemann S. Molecular epidemiology of pulmonary tuberculosis in Belgrade, central Serbia. J Clin Microbiol. 2003;41:4372-7. http://dx.doi.org/ 10.1128/JCM.41.9.4372-4377.2003

4. Merker M, Blin C, Mona S, Duforet-Frebourg N, Lecher S, Willery E, et al. Evolutionary history and global spread of the Mycobacterium tuberculosis Beijing lineage. Nat Genet. 2015; 47:242-9. http://dx.doi.org/10.1038/ng.3195

5. Coll F, McNerney R, Guerra-Assunção JA, Glynn JR, Perdigão J, Viveiros M, et al. A robust SNP barcode for typing Mycobacterium tuberculosis complex strains. Nat Commun. 2014;5:4812. http://dx.doi.org/10.1038/ncomms5812

6. Niemann S, Merker M, Kohl T, Supply P. Impact of genetic diversity on the biology of Mycobacterium tuberculosis complex strains. Microbiol Spectr. 2016;4:TBTB2-0022-2016.

7. Comas I, Borrell S, Roetzer A, Rose G, Malla B, Kato-Maeda M, et al. Whole-genome sequencing of rifampicin-resistant Mycobacterium tuberculosis strains identifies compensatory mutations in RNA polymerase genes. Nat Genet. 2012;44:106-10. http://dx.doi.org/10.1038/ng.1038

8. Jovanovic D, Skodric-Trifunovic V, Markovic-Denic L, Stevic R, Vlajinac H. Clinical and epidemiological evaluation of tuberculosis in Serbia, 1990-2004. Int J Tuberc Lung Dis. 2007;11:647-51

9. Pachi A, Bratis D, Moussas G, Tselebis A. Psychiatric morbidity and other factors affecting treatment adherence in pulmonary tuberculosis patients. Tuberc Res Treat. 2013;2013:489865. http://dx.doi.org/10.1155/2013/489865

10. Tosevski DL, Gajic SD, Milovancevic MP. Mental healthcare in Serbia. Int Psychiatry. 2010;7:13-5. http://dx.doi.org/10.1192/ S1749367600000941

Address for correspondence: Irena Arandjelović, University of Belgrade Institute of Microbiology and Immunology, Faculty of Medicine, dr Subotica 1, 11000 Belgrade, Serbia; email: irena.arandjelovic@med.bg.ac.rs

\section{EID Podcast:}

Visions of Matchstick Men and Icons of Industrialization

Byron Breedlove, managing editor of the journal, discusses and reads his November 2017 cover art essay. This cover (Going to Work, 1943) is by English artist Laurence Stephen Lowry (1887-1976) who died of pneumonia in 1976.

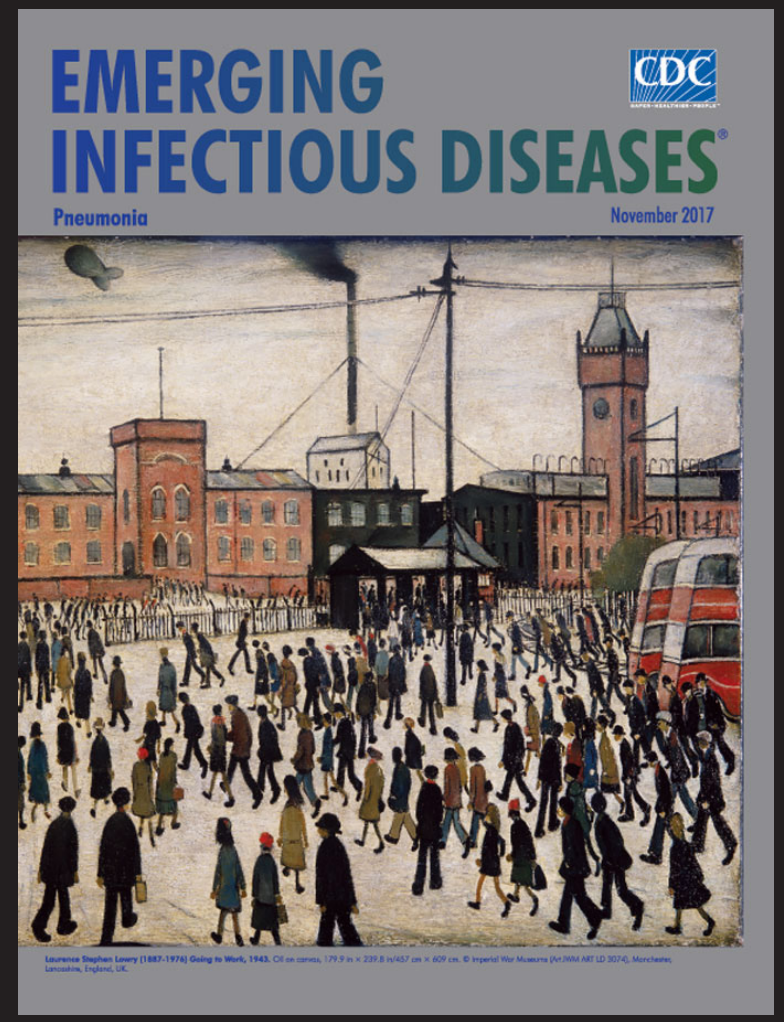

Visit our website to listen: https:/ / www2c.cdc.gov/ podcasts/player.asp?f $=\mathbf{8 6 4 7 1 7 3}$ EMERGING INFECTIOUS DISEASES 